\title{
Exact confidence intervals of the extended Orey index for Gaussian processes
}

\author{
K. Kubilius ${ }^{1, *, \dagger}$ and D. Melichov ${ }^{2}$ \\ ${ }^{1}$ Vilnius University, Institute of Mathematics and Informatics, Akademijos 4, \\ LT-08663, Vilnius, Lithuania \\ ${ }^{2}$ Vilnius Gediminas Technical University, Saulètekio al. 11, LT-10223, Vilnius, \\ Lithuania
}

\begin{abstract}
In this paper exact confidence intervals for the Orey index of Gaussian processes are obtained using concentration inequalities for Gaussian quadratic forms and discrete observations of the underlying process. The obtained result is applied to Gaussian processes with the Orey index which not necessarily have stationary increments.
\end{abstract}

Keywords: concentration inequality, confidence intervals, Gaussian processes with the Orey index, fractional Ornstein-Uhlenbeck process, sub-fractional Brownian motion

AMS Subject Classification: primary 60G15; secondary 60F05, 60H07.

\section{Introduction}

Let $X=\{X(t): t \in[0, T]\}$ be a second order stochastic process with the incremental variance function $\sigma_{X}^{2}$ defined on $[0, T]^{2}:=[0, T] \times[0, T]$ with values

$$
\sigma_{X}^{2}(s, t):=\mathbf{E}[X(t)-X(s)]^{2}, \quad(s, t) \in[0, T]^{2} .
$$

Denote by $\widetilde{\Psi}$ a class of continuous functions $\varphi:(0, T] \rightarrow[0, \infty)$ such that $\lim _{h \downarrow 0} \varphi(h)=0$ and $L(h)=\varphi(h) / h \rightarrow \infty, h \downarrow 0$. For example, we can take $\varphi(h)=h|\ln h|$ or $\varphi(h)=h^{1-\varepsilon}$ for $0<\varepsilon<1$. Set

$$
\begin{aligned}
& \gamma_{*}:=\inf \left\{\gamma>0: \lim _{h \downarrow 0} \sup _{\varphi(h) \leqslant s \leqslant T-h} \frac{h^{\gamma}}{\sigma_{X}(s, s+h)}=0\right\}, \\
& \widetilde{\gamma}_{*}:=\inf \left\{\gamma>0: \quad \lim _{h \downarrow 0} \frac{h^{\gamma}}{\sigma_{X}(0, h)}=0\right\}
\end{aligned}
$$

and

$$
\begin{aligned}
& \gamma^{*}:=\sup \left\{\gamma>0: \lim _{h \downarrow 0} \inf _{\varphi(h) \leqslant s \leqslant T-h} \frac{h^{\gamma}}{\sigma_{X}(s, s+h)}=+\infty\right\}, \\
& \widetilde{\gamma}^{*}:=\sup \left\{\gamma>0: \lim _{h \downarrow 0} \frac{h^{\gamma}}{\sigma_{X}(0, h)}=+\infty\right\},
\end{aligned}
$$

where $\varphi \in \widetilde{\Psi}$. Note that $0 \leqslant \widetilde{\gamma}^{*} \leqslant \widetilde{\gamma}_{*} \leqslant+\infty$ and $0 \leqslant \gamma^{*} \leqslant \gamma_{*} \leqslant+\infty$. In paper [13] we used a narrower class of functions $\Psi$, i.e. such functions which additionally satisfy condition $\lim _{h \downarrow 0}\left[h \cdot L^{3}(h)\right]=0$. This condition is not necessary for the existence of the Orey index. It is required only for consideration of almost sure asymptotic behavior of the second-order quadratic variations of Gaussian processes.

We give the following extension of the Orey index.

*Corresponding author. E-mail: kestutis.kubilius@mii.vu.lt

$\dagger$ This research was funded by a grant (No. MIP-048/2014) from the Research Council of Lithuania. 
Definition 1 ([13]) Let $X=\{X(t): t \in[0, T]\}$ be a second order stochastic process with the incremental variance function $\sigma_{X}^{2}$ such that $\sup _{0 \leqslant s \leqslant T-h} \sigma_{X}(s, s+h) \rightarrow 0$ as $h \rightarrow 0$. If $\gamma_{*}=\widetilde{\gamma}_{*}=\gamma^{*}=\widetilde{\gamma}^{*}$ for any function $\varphi \in \widetilde{\Psi}$, then we say that the process $X$ has the Orey index $\gamma_{X}=\gamma_{*}=\widetilde{\gamma}_{*}=\gamma^{*}=\widetilde{\gamma}^{*}$.

Assume that for some $\gamma \in(0,1)$ the second order stochastic process $X$ satisfies conditions:

(C1) $\sigma_{X}(0, \delta) \asymp \delta^{\gamma}$, i.e., $\sigma_{X}(0, \delta)$ and $\delta^{\gamma}$ are of the same order as $\delta \downarrow 0$;

(C2) there exist a constant $\varkappa>0$ such that

$$
\Lambda(\delta):=\sup _{\varphi(\delta) \leqslant t \leqslant T-\delta} \sup _{0<h \leqslant \delta}\left|\frac{\sigma_{X}(t, t+h)}{\varkappa h^{\gamma}}-1\right| \longrightarrow 0 \quad \text { as } \delta \downarrow 0
$$

for every function $\varphi \in \widetilde{\Psi}$.

If for some constant $\gamma \in(0,1)$ the second order stochastic process $X$ satisfies conditions $(C 1)$ and $(C 2)$, then the Orey index is equal to $\gamma$ (see [13]).

Recently much attention has been given to studies and applications of Gaussian processes such as fractional Brownian motion (fBm), sub-fractional Brownian motion (subfBm), bifractional Brownian motion (bifBm), fractional Ornstein-Uhlenbeck process. All of them are Gaussian processes and they have the Orey indexes. Consequently, examining Gaussian processes with the Orey index we thus examine the processes listed above.

Many authors (see [9, [10, [12, 8, 7], 1], 2], [17, 15]) considered an almost sure convergence and asymptotic normality of the generalized quadratic variations associated to the filter $a$ (see [12]) of a wide class of processes with Gaussian increments. The strong consistency of the Orey index estimator was proven in [13].

In the papers of Breton et al. [4] and Breton and Coeurjolly [5], an exact (non-asymptotic) confidence interval for the Hurst index of $\mathrm{fBm}$ was derived with the aid of concentration inequalities for quadratic forms of Gaussian process. The obtained confidence intervals for the Hurst parameter were based on a single observation of a discretized sample path of the interval $[0,1]$ of fBm. Exact confidence intervals for sub-fractional Brownian motion were considered in 14 but are not sufficiently precise.

The purpose of article is to extend the results of Breton et al. 4] and Breton and Coeurjolly [5] as well as to apply them to Gaussian processes with the Orey index which may not have stationary increments.

The paper is organized in the following way. In Section 2 we give exact confidence intervals for the Orey index of Gaussian process. Section 3 contains some application results for known Gaussian processes which may not have stationary increments. Finally, in Section 4 some simulations are given in order to illustrate the obtained results. In addition, Appendix includes the $\mathrm{R}$ code listings of simulations.

\section{Confidence intervals}

First, we formulate a concentration inequality for a family of Gaussian r.v.'s. Consider a finite centered Gaussian family $X=\left\{X_{k}: k=1, \ldots, M\right\}$, and write $d_{k j}=\mathbf{E}\left(X_{k} X_{j}\right)$. Define two quadratic forms associated with $X$ and with some real coefficient $c$ :

$$
Q_{1}(c, X)=c \sum_{k=1}^{M}\left(X_{k}^{2}-d_{k k}\right), \quad Q_{2}(c, X)=2 c^{2} \sum_{k, j=1}^{M} X_{k} X_{j} d_{k j} .
$$

The following statement characterizes the tail behavior of $Q_{1}(c, X)$.

Theorem 2 ([4, [5]) Suppose that $Q_{1}(c, X)$ is not a.s. zero and fix $\alpha \geq 0$ and $\beta>0$. Assume that $Q_{2}(c, X) \leq \alpha Q_{1}(c, X)+\beta$, a.s.-P. Then, for all $z>0$, we have

$$
\begin{gathered}
\mathbf{P}\left(Q_{1}(c, X) \geq z\right) \leqslant \varphi_{r}(z ; \alpha, \beta):=e^{-z / \alpha}\left(1+\frac{\alpha z}{\beta}\right)^{\beta / \alpha^{2}} \\
\mathbf{P}\left(Q_{1}(c, X) \leq-z\right) \leqslant \varphi_{l}(z ; \alpha, \beta):=e^{z / \alpha}\left(1-\frac{\alpha z}{\beta}\right)^{\beta / \alpha^{2}} \mathbf{1}_{[0, \beta / \alpha]}(z) .
\end{gathered}
$$

Remark 3 [5] Note that $\varphi_{r}(\cdot ; \alpha, \beta)$ (resp. $\left.\varphi_{l}(\cdot ; \alpha, \beta)\right)$ is a bijective function from $(0,+\infty)$ (resp. $(0, \beta / \alpha))$ to $(0,1)$. 
Next, we apply the obtained concentration inequality to second order quadratic variations.

Let $X$ be a centered Gaussian process satisfying conditions $(C 1)$ and $(C 2)$ with the Orey index $\gamma \in(0,1)$. Denote $Y=\left(Y_{1, n}, \ldots, Y_{n-1, n}\right)$, where

$$
Y_{k, n}=\frac{n^{\gamma}}{T^{\gamma} \varkappa \sqrt{4-2^{2 \gamma}}} \Delta_{k, n}^{(2)} X, \quad \Delta_{k, n}^{(2)} X=X\left(t_{k+1}^{n}\right)-2 X\left(t_{k}^{n}\right)+X\left(t_{k-1}^{n}\right)
$$

$t_{k}^{n}=\frac{k T}{n}$ and $\varkappa$ is a constant defined in condition $(C 2)$. Set $d_{j k}^{Y, n}=\mathbf{E} Y_{j, n} Y_{k, n}$.

Proposition 4 Assume that there exists a sequence of real numbers $\left(\varepsilon_{n}\right)$ not depending on $\gamma$ and such that

$$
\left|\frac{1}{n-1} \sum_{k=1}^{n-1} d_{k k}^{Y, n}-1\right| \leqslant \varepsilon_{n}, \quad \varepsilon_{n} \downarrow 0 .
$$

Then for all $z>0$ we have

$$
\begin{gathered}
\mathbf{P}\left(\frac{1}{\sqrt{n-1}} \sum_{k=1}^{n-1}\left[\left(Y_{k, n}\right)^{2}-d_{k k}^{Y, n}\right] \geqslant z\right) \leqslant \varphi_{r, n}\left(z ; \nu_{n}, \varepsilon_{n}\right), \\
\mathbf{P}\left(\frac{1}{\sqrt{n-1}} \sum_{k=1}^{n-1}\left[\left(Y_{k, n}\right)^{2}-d_{k k}^{Y, n}\right] \leqslant-z\right) \leqslant \varphi_{l, n}\left(z ; \nu_{n}, \varepsilon_{n}\right),
\end{gathered}
$$

where

$$
\begin{aligned}
\varphi_{r, n}\left(z ; \nu_{n}, \varepsilon_{n}\right) & :=\exp \left\{-\frac{z \sqrt{n-1}}{2 \nu_{n}}\right\}\left(1+\frac{z}{\left(\varepsilon_{n}+1\right) \sqrt{n-1}}\right)^{\frac{\left(\varepsilon_{n}+1\right)(n-1)}{2 \nu_{n}}} \\
\varphi_{l, n}\left(z ; \nu_{n}, \varepsilon_{n}\right) & :=\exp \left\{\frac{z \sqrt{n-1}}{2 \nu_{n}}\right\}\left(1-\frac{z}{\left(\varepsilon_{n}+1\right) \sqrt{n-1}}\right)^{\frac{\left(\varepsilon_{n}+1\right)(n-1)}{2 \nu_{n}}} \mathbf{1}_{\left[0,\left(\varepsilon_{n}+1\right) \sqrt{n-1}\right]}(z), \\
\nu_{n} & =\max _{1 \leqslant k \leqslant n-1} \sum_{j=1}^{n-1}\left|d_{k j}^{Y, n}\right| .
\end{aligned}
$$

Proof. Denote

$$
Q_{1}\left((n-1)^{-1 / 2}, Y\right)=\frac{1}{\sqrt{n-1}} \sum_{k=1}^{n-1}\left[\left(Y_{k, n}\right)^{2}-d_{k k}^{Y, n}\right]
$$

and

$$
Q_{2}\left((n-1)^{-1 / 2}, Y\right)=\frac{2}{n-1} \sum_{k, j=1}^{n-1} Y_{k} Y_{j} d_{k j}^{Y, n}
$$

Then

$$
\begin{aligned}
& Q_{2}\left((n-1)^{-1 / 2}, Y\right) \\
& \quad \leqslant \frac{2}{n-1} \sum_{k, j=1}^{n-1}\left|Y_{k}\right| \cdot\left|Y_{j}\right| \cdot\left|d_{k j}^{Y, n}\right| \leqslant \frac{1}{n-1} \sum_{k, j=1}^{n-1}\left[Y_{k}^{2}+Y_{j}^{2}\right] \cdot\left|d_{k j}^{Y, n}\right| \\
& \quad=\frac{2}{n-1} \sum_{k, j=1}^{n-1} Y_{k}^{2}\left|d_{k j}^{Y, n}\right|=\frac{2}{n-1} \sum_{k=1}^{n-1} Y_{k}^{2} \sum_{j=1}^{n-1}\left|d_{k j}^{Y, n}\right| \\
& \quad \leqslant \frac{2}{n-1} \sum_{k=1}^{n-1} Y_{k}^{2}\left(\max _{1 \leqslant k \leqslant n-1} \sum_{j=1}^{n-1}\left|d_{k j}^{Y, n}\right|\right) \\
& \quad=\frac{2 \nu_{n}}{\sqrt{n-1}}\left(Q_{1}\left((n-1)^{-1 / 2}, Y\right)+\frac{1}{\sqrt{n-1}} \sum_{k=1}^{n-1}\left[d_{k k}^{Y, n}-1\right]+\sqrt{n-1}\right) \\
& \quad \leqslant \alpha_{n} Q_{1}\left((n-1)^{-1 / 2}, Y\right)+\beta_{n},
\end{aligned}
$$

where

$$
\alpha_{n}=\frac{2 \nu_{n}}{\sqrt{n-1}}, \quad \beta_{n}=2 \nu_{n}\left(\varepsilon_{n}+1\right) .
$$


Thus

$$
\begin{aligned}
& \mathbf{P}\left(Q_{1}\left((n-1)^{-1 / 2}, Y\right) \geqslant z\right) \leqslant \varphi_{r, n}\left(z ; \nu_{n}, \varepsilon_{n}\right) \\
& \mathbf{P}\left(Q_{1}\left((n-1)^{-1 / 2}, Y\right) \leqslant-z\right) \leqslant \varphi_{l, n}\left(z ; \nu_{n}, \varepsilon_{n}\right)
\end{aligned}
$$

and the proof is completed.

Remark 5 Since $\varphi_{l, n}\left(z ; \cdot, \varepsilon_{n}\right)$ and $\varphi_{r, n}\left(z ; \cdot, \varepsilon_{n}\right)$ are non-decreasing, inequalities (3) and (4) remain true with $\nu_{n}$ replacing $v_{n}$, where $\nu_{n} \leqslant v_{n}$.

For any $\alpha \in(0,1)$ and $v_{n}\left(\nu_{n} \leqslant v_{n}\right)$, denote by $q_{l, n}(\alpha):=\left(\varphi_{l, n}\right)^{-1}\left(\alpha ; v_{n}, \varepsilon_{n}\right)$ and $q_{r, n}(\alpha):=$ $\left(\varphi_{r, n}\right)^{-1}\left(\alpha ; v_{n}, \varepsilon_{n}\right)$. For convenience we define

$$
x_{l, n-1}(\alpha):=1-\frac{q_{l, n-1}(\alpha)}{\sqrt{n-1}} \quad \text { and } \quad x_{r, n-1}(\alpha):=1+\frac{q_{r, n-1}(\alpha)}{\sqrt{n-1}} .
$$

Note that Remark 3 above ensures that for any $\alpha \in(0,1)$ and for all $n>1$, we have $x_{l, n-1}(\alpha)>$ 0 . Set

$$
S_{n}:=\frac{1}{n-1} \sum_{k=1}^{n-1}\left(\Delta_{k, n}^{(2)} X\right)^{2}, \quad g_{n, T}(x):=2 x \ln (n / T)-\ln \left(4-2^{2 x}\right), \quad x \in(0,1),
$$

and

$$
\ln ^{*} x=\left\{\begin{array}{lll}
-\infty & \text { if } & x=0 \\
\ln x & \text { if } & x>0
\end{array}\right.
$$

The function $g_{n, T}(x)$ is a strictly increasing bijection from $(0,1)$ to $(-\ln 3,+\infty)$ if $n>T$.

Theorem 6 Let $\alpha \in(0,1)$. Assume that conditions of Proposition 4 are satisfied and there are constants $v_{n}$ such that $\nu_{n} \leqslant v_{n}$. Then

$$
\mathbb{P}\left(\gamma \in\left[\gamma_{n}^{\text {inf }}(\alpha), \gamma_{n}^{\text {sup }}(\alpha)\right]\right) \geqslant 1-\alpha
$$

where

$$
\begin{aligned}
& \gamma_{n}^{\text {inf }}(\alpha):=\max \left(0, g_{n, T}^{-1}\left[\max \left(\ln ^{*}\left(\left(\frac{x_{l, n-1}(\alpha / 2)-\varepsilon_{n}}{S_{n}} \varkappa^{2}\right) \vee 0\right),-\ln 3\right)\right],\right. \\
& \gamma_{n}^{\text {sup }}(\alpha):=\min \left(1, g_{n, T}^{-1}\left[\ln \left(\frac{x_{r, n-1}(\alpha / 2)+\varepsilon_{n}}{S_{n}} \varkappa^{2}\right)\right]\right) .
\end{aligned}
$$

Proof. Denote

$$
Z_{n}=(n-1)^{-1 / 2} V_{n}^{(2)}(Y, 2)-\sqrt{n-1}=\sqrt{n-1}\left[(n-1)^{-1} V_{n}^{(2)}(Y, 2)-1\right]
$$

where

$$
V_{n}^{(2)}(Y, 2)=\sum_{i=1}^{n-1}\left(\Delta_{k, n}^{(2)} Y\right)^{2}
$$

Then

$$
\begin{aligned}
\{ & \left.-q_{l, n-1}(\alpha / 2) \leqslant(n-1)^{-1 / 2} \sum_{k=1}^{n-1}\left[\left(Y_{k, n}\right)^{2}-d_{k k}^{Y, n}\right] \leqslant q_{r, n-1}(\alpha / 2)\right\} \\
& =\left\{-q_{l, n-1}(\alpha / 2)+(n-1)^{-1 / 2} \sum_{k=1}^{n-1}\left[d_{k k}^{Y, n}-1\right] \leqslant Z_{n} \leqslant q_{r, n-1}(\alpha / 2)+(n-1)^{-1 / 2} \sum_{k=1}^{n-1}\left[d_{k k}^{Y, n}-1\right]\right\} \\
& =\left\{x_{l, n-1}(\alpha / 2)+(n-1)^{-1} \sum_{k=1}^{n-1}\left[d_{k k}^{Y, n}-1\right] \leqslant \frac{n^{2 \gamma}}{T^{2 \gamma} \varkappa^{2}\left(4-4^{\gamma}\right)} S_{n}\right. \\
& \subset\left\{x_{l, n-1}(\alpha / 2)-\varepsilon_{n} \leqslant \frac{n^{2 \gamma}}{T^{2 \gamma} \varkappa^{2}\left(4-2^{2 \gamma}\right)} S_{n} \leqslant x_{r, n-1}(\alpha / 2)+\varepsilon_{n}\right\} \\
= & \left\{\left(x_{l, n-1}(\alpha / 2)-\varepsilon_{n}\right) \vee 0 \leqslant \frac{n^{2 \gamma}}{T^{2 \gamma} \varkappa^{2}\left(4-2^{2 \gamma}\right)} S_{n} \leqslant x_{r, n-1}(\alpha / 2)+\varepsilon_{n}\right\} \\
= & \left\{\ln ^{*}\left(\left(x_{l, n-1}(\alpha / 2)-\varepsilon_{n}\right) \vee 0\right)-\ln S_{n}+\ln \varkappa^{2} \leqslant g_{n, T}(\gamma) \leqslant \ln \left(x_{r, n-1}(\alpha / 2)+\varepsilon_{n}\right)-\ln S_{n}+\ln \varkappa^{2}\right\} \\
= & \left\{\left[\ln ^{*}\left(\frac{\left(x_{l, n-1}(\alpha / 2)-\varepsilon_{n}\right) \vee 0}{S_{n}} \varkappa^{2}\right)\right] \vee(-\ln 3) \leqslant g_{n, T}(\gamma) \leqslant \ln \left(\frac{x_{r, n-1}(\alpha / 2)+\varepsilon_{n}}{S_{n}} \varkappa^{2}\right)\right.
\end{aligned}
$$


Note that

$$
\mathbf{P}\left(-q_{l, n-1}(\alpha / 2) \leqslant \frac{1}{\sqrt{n-1}} \sum_{k=1}^{n-1}\left[\left(Y_{k, n}\right)^{2}-d_{k k}^{Y, n}\right] \leqslant q_{r, n-1}(\alpha / 2)\right) \geqslant 1-\alpha
$$

Thus

$$
\mathbf{P}\left(\gamma \in\left[\gamma_{n}^{\text {inf }}(\alpha), \gamma_{n}^{\text {sup }}(\alpha)\right]\right) \geqslant 1-\alpha
$$

The proof is completed.

\section{Applications}

In this section we obtain the confidence intervals for subfBm, bifBm and the fractional Ornstein-Uhlenbeck process. For this purpose we apply the Theorem 6 In order to apply the Theorem 6, it suffices to find the sequence of real numbers $\left(\varepsilon_{n}\right)$ in the estimation $(2)$ and estimate $\nu_{n}$. In the considered cases as the special case appears the Brownian motion. We exclude it from consideration in view of its properties (in particular, independent increaments). It is easy to see that

$$
\frac{n}{2 T} d_{k k}^{B, n}=1, \quad \frac{n}{2 T} \max _{1 \leqslant k \leqslant n-1} \sum_{j=1}^{n-1}\left|d_{j k}^{B, n}\right| \leqslant 2,
$$

where $d_{j k}^{B, n}=\mathbf{E} \Delta_{j, n}^{(2)} B \Delta_{k, n}^{(2)} B$ and $B$ is Brownian motion.

\subsection{Sub-fractional Brownian motion}

Definition 7 ([3]) A sub-fractional Brownian motion (subfBm) with the index $H, H \in$ $(0,1)$, is a mean zero Gaussian stochastic process $S^{H}=\left(S_{t}^{H}, t \geqslant 0\right)$ with the covariance function

$$
G_{H}(s, t):=s^{2 H}+t^{2 H}-\frac{1}{2}\left[(s+t)^{2 H}+|s-t|^{2 H}\right] .
$$

The case $H=1 / 2$ corresponds to the Brownian motion. For $H \neq 1 / 2$ this process has some of the main properties of $\mathrm{fBm}$, but its increments are not stationary.

The incremental variance function of subfBm is of the following form

$$
\sigma_{S^{H}}^{2}(s, t)=\mathbf{E}\left|S_{t}^{H}-S_{s}^{H}\right|^{2}=|t-s|^{2 H}+(s+t)^{2 H}-2^{2 H-1}\left(t^{2 H}+s^{2 H}\right) .
$$

For any $0 \leqslant s \leqslant t \leqslant T$ the inequalities (see [3])

$$
\begin{array}{ll}
(t-s)^{2 H} \leqslant \sigma_{S^{H}}^{2}(s, t) \leqslant\left(2-2^{2 H-1}\right)(t-s)^{2 H}, & \text { if } \quad 0<H<1 / 2, \\
\left(2-2^{2 H-1}\right)(t-s)^{2 H} \leqslant \sigma_{S^{H}}^{2}(s, t) \leqslant(t-s)^{2 H}, & \text { if } \quad 1 / 2<H<1
\end{array}
$$

hold.

It is known that for subfBm the Orey index is equal to $H$ (see [13]). Now we prove the following lemma.

Lemma 8 Assume that $S^{H}=\left\{S^{H}(t): t \in[0, T]\right\}$ is a subfBm. If $H \neq 1 / 2$ then

$$
\left|\frac{1}{n-1} \sum_{k=1}^{n-1} d_{k k}^{S^{H}, n}-1\right| \leqslant\left(\frac{T}{n}\right)^{2 / 3}\left(\frac{1}{6 T} \frac{n}{n-1}+\frac{33}{9 \ln 4}\right)
$$

where

$$
d_{k k}^{S^{H}}{ }^{n}=\frac{n^{2 H}}{T^{2 H}\left(4-2^{2 H}\right)} \mathbf{E}\left(\Delta_{k, n}^{(2)} S^{H}\right)^{2}
$$

Proof. Observe that the following equality

$$
\begin{aligned}
\mathbf{E}\left(S_{t+h}^{H}-2 S_{t}^{H}+S_{t-h}^{H}\right)^{2}= & \left(4-2^{2 H}\right) h^{2 H}-2^{2 H-1}(t+h)^{2 H}-3 \cdot 2^{2 H} t^{2 H} \\
& -2^{2 H-1}(t-h)^{2 H}+2(2 t+h)^{2 H}+2(2 t-h)^{2 H}
\end{aligned}
$$


holds. Thus

$$
\begin{aligned}
d_{k k}^{S^{H}, n} & =1-\frac{2^{2 H-1}(k+1)^{2 H}+3 \cdot 2^{2 H} k^{2 H}+2^{2 H-1}(k-1)^{2 H}-2(2 k+1)^{2 H}-2(2 k-1)^{2 H}}{4-2^{2 H}} \\
& =1-\frac{b(k, H)}{4-2^{2 H}} .
\end{aligned}
$$

For simplicity we shall omit the index $n$ for $d_{k k}^{S^{H}, n}$. Using computer modeling we obtain the inequalities

$$
\max _{H \in(0,1)} d_{k k}^{S^{H}} \leqslant \max _{H \in(0,1)} d_{11}^{S^{H}}=\frac{7}{6} \quad \text { and } \max _{H \in(0,1)} \frac{|b(k, H)|}{4-2^{2 H}} \leqslant \max _{H \in(0,1)} \frac{|b(1, H)|}{4-2^{2 H}} \leqslant \frac{1}{6} .
$$

Let $\varphi \in \widetilde{\Psi}$ and denote $\tau_{n}:=\left[\varphi\left(T n^{-1}\right) n T^{-1}\right]$, where $[a]$ is an integer part of $a$. Then

$$
\begin{aligned}
\left|\frac{1}{n-1} \sum_{k=1}^{n-1} d_{k k}^{S^{H}}-1\right| & \leqslant \frac{1}{n-1} \sum_{k=1}^{\tau_{n}} \frac{|b(k, H)|}{4-2^{2 H}}+\max _{\tau_{n}+1 \leqslant k \leqslant n-1} \frac{|b(k, H)|}{4-2^{2 H}} \\
& =I_{1}+I_{2} .
\end{aligned}
$$

It is clear that

$$
I_{1} \leqslant \frac{\tau_{n}}{n-1} \max _{1 \leqslant k \leqslant \tau_{n}} \frac{|b(k, H)|}{4-2^{2 H}} \leqslant \frac{\tau_{n}}{n-1} \frac{|b(1, H)|}{4-2^{2 H}} \leqslant \frac{1}{6} \varphi\left(T n^{-1}\right) T^{-1} \frac{n}{n-1} .
$$

Now we estimate $b(k, H)$ for $k \geqslant 2$. Using the formula

$$
(1+x)^{\alpha}=1+\sum_{k=1}^{\infty} \frac{\alpha(\alpha-1) \cdots(\alpha-k+1)}{k !} x^{k} \quad \text { for }-1<x<1,
$$

we obtain

$$
\begin{aligned}
b(k, H) & =2^{2 H-1}(k+1)^{2 H}+3 \cdot 2^{2 H} k^{2 H}+2^{2 H-1}(k-1)^{2 H}-2(2 k+1)^{2 H}-2(2 k-1)^{2 H} \\
& =2^{2 H-1} k^{2 H}\left[\left(1+\frac{1}{k}\right)^{2 H}+6+\left(1-\frac{1}{k}\right)^{2 H}-4\left(1+\frac{1}{2 k}\right)^{2 H}-4\left(1-\frac{1}{2 k}\right)^{2 H}\right] \\
& =2^{2 H-1} k^{2 H}\left[\sum_{m=1}^{\infty} \frac{2 H(2 H-1) \cdots(2 H-m+1)}{m !}\left(\frac{1}{k}\right)^{m}\left[1+(-1)^{m}-2^{2-m}-(-1)^{m} 2^{2-m}\right]\right] \\
& =2^{2 H} k^{2 H}\left[\sum_{m=2}^{\infty} \frac{2 H(2 H-1) \cdots(2 H-2 m+1)}{(2 m) !}\left(\frac{1}{k}\right)^{2 m}\left(1-2^{2-2 m}\right)\right] .
\end{aligned}
$$

Note that the sign of $2 H(2 H-1)(2 H-2) \cdots(2 H-2 m+1)$ is the same as that of $2 H-1$. Thus

$$
\begin{aligned}
\frac{|b(k, H)|}{4-2^{2 H}} & \leqslant \frac{2^{2 H} k^{2 H}}{4-2^{2 H}}\left[\sum_{m=2}^{\infty} \frac{2 H|2 H-1|(2-2 H) \cdots(2 m-1-2 H)}{(2 m) !}\left(\frac{1}{k}\right)^{2 m}\left[1-2^{2-2 m}\right]\right] \\
& \leqslant \frac{3 \cdot 2^{2 H} k^{2 H}}{4 \ln 4} \sum_{m=2}^{\infty} \frac{2(2 m-1) !}{(2 m) !}\left(\frac{1}{k}\right)^{2 m} \\
& \leqslant \frac{3 \cdot 2^{2 H}}{4 \ln 4} k^{2 H} \sum_{m=2}^{\infty} \frac{1}{m}\left(\frac{1}{k^{2}}\right)^{m} \leqslant \frac{3 \cdot 2^{2 H}}{4 \ln 4} k^{2 H-2} \sum_{m=2}^{\infty} \frac{1}{m-1}\left(\frac{1}{k^{2}}\right)^{m-1} \\
& =-\frac{3 \cdot 2^{2 H}}{4 \ln 4} k^{2 H-2} \ln \left(1-\frac{1}{k^{2}}\right) \leqslant \frac{33 \cdot 2^{2 H}}{36 \ln 4} k^{2 H-4} \leqslant \frac{33}{9 \ln 4} k^{-2}
\end{aligned}
$$

since

$$
\begin{aligned}
& 2 H|1-2 H|(2-2 H) \cdots(2 m-1-2 H) \leqslant 2(1-H)(2 m-1) ! \\
& \log (1-u)=-\sum_{k=1}^{\infty} \frac{u^{k}}{k} \quad \text { if } 0 \leq u<1 \quad \text { and } \quad-\log (1-u) \leqslant \frac{11}{9} u \quad \text { if } 0 \leq u \leqslant 1 / 4, \\
& (1-H) \ln 4<4-2^{2 H} .
\end{aligned}
$$


So

$$
\begin{aligned}
\left|\frac{1}{n-1} \sum_{k=1}^{n-1} d_{k k}^{S^{H}}-1\right| & \leqslant \frac{1}{6} \varphi\left(T n^{-1}\right) T^{-1} \frac{n}{n-1}+\frac{33}{9 \ln 4} \frac{1}{\left(\tau_{n}+1\right)^{2}} \\
& \leqslant \frac{1}{6} \varphi\left(T n^{-1}\right) T^{-1} \frac{n}{n-1}+\frac{33}{9 \ln 4} \frac{T^{2}}{\varphi^{2}\left(T n^{-1}\right) n^{2}} .
\end{aligned}
$$

Let $\varphi\left(T n^{-1}\right)=\left(T n^{-1}\right)^{2 / 3}$. It belongs to the class of functions $\widetilde{\Psi}$. After putting into the obtained inequality we get

$$
\left|\frac{1}{n-1} \sum_{k=1}^{n-1} d_{k k}^{S^{H}}-1\right| \leqslant \frac{1}{6} \frac{1}{T^{1 / 3} n^{2 / 3}} \frac{n}{n-1}+\frac{33}{9 \ln 4}\left(\frac{T}{n}\right)^{2 / 3}=\left(\frac{T}{n}\right)^{2 / 3}\left(\frac{2}{15 T} \frac{n}{n-1}+\frac{33}{9 \ln 4}\right) .
$$

Lemma 9 Assume that $S^{H}=\left\{S_{t}^{H}: t \in[0, T]\right\}$ is a subfBm. If $H \neq 1 / 2$ then

$$
\max _{1 \leqslant k \leqslant n-1} \sum_{j=1}^{n-1}\left|d_{j k}^{S^{H}, n}\right| \leqslant \frac{9}{2}
$$

where

$$
d_{j k}^{S^{H}, n}=\frac{n^{2 H}}{T^{2 H}\left(4-2^{2 H}\right)} \widehat{d}_{j k}^{S^{H}, n}, \quad \widehat{d}_{j k}^{S^{H}, n}=\mathbf{E} \Delta_{j, n}^{(2)} S^{H} \Delta_{k, n}^{(2)} S^{H} .
$$

Proof. The fourth order mixed partial derivative of the covariance function $G_{H}(s, t)$ is of the following form

$$
\frac{\partial^{4} G_{H}}{\partial s^{2} \partial t^{2}}(s, t)=-C_{H}\left[\frac{1}{|s-t|^{2(2-H)}}+\frac{1}{(s+t)^{2(2-H)}}\right]
$$

for each $s, t>0$ such that $s \neq t$, where $C_{H}=H(2 H-1)(2 H-2)(2 H-3)$. Since the covariance function $G_{H}(s, t)$ is continuous in $[0, T]^{2}$ and the derivative $\frac{\partial^{4} R}{\partial s^{2} \partial t^{2}}$ is continuous in $(0, T]^{2} /\{s=t\}$ then for $H \neq 1 / 2$ and $j \neq 1$ or $k \neq 1$

$$
\widehat{d}_{j k}^{S H}, n=\int_{t_{j}^{n}}^{t_{j+1}^{n}} d u \int_{u-T / n}^{u} d v \int_{t_{k}^{n}}^{t_{k+1}^{n}} d x \int_{x-T / n}^{x} \frac{\partial^{4} G_{H}}{\partial s^{2} \partial t^{2}}(v, y) d y .
$$

Assume that $R_{H}$ is the covariance function of the $\mathrm{fBm} B^{H}$. Then the derivative

$$
\frac{\partial^{4} R_{H}}{\partial s^{2} \partial t^{2}}(s, t)=-\frac{C_{H}}{|s-t|^{4-2 H}}
$$

of the covariance function $R_{H}$ is continuous in $(0, T]^{2} /\{s=t\}$ and

$$
\begin{aligned}
\widehat{d}_{j k}^{B H}, n & :=\mathbf{E}\left(\Delta_{k, n}^{(2)} B^{H}\right)^{2}=-\int_{t_{j}^{n}}^{t_{j+1}^{n}} d u \int_{u-T / n}^{u} d v \int_{t_{k}^{n}}^{t_{k+1}^{n}} d x \int_{x-T / n}^{x} \frac{C_{H}}{(v-y)^{4-2 H}} d y \\
& =\rho_{H}(j-k) \frac{T^{2 H}}{n^{2 H}}
\end{aligned}
$$

where

$$
\rho_{H}(r)=\frac{1}{2}\left(-|r-2|^{2 H}+4|r-1|^{2 H}-6|r|^{2 H}+4|r+1|^{2 H}-|r+2|^{2 H}\right), \quad r \in \mathbb{N} \cup\{0\} .
$$

Note that $(s+t) \geqslant|s-t|$ and

$$
\begin{aligned}
\left|\widehat{d}_{j k}^{S H}, n-\widehat{d}_{j k}^{B H}, n\right| & =\left|\int_{t_{j}^{n}}^{t_{j+1}^{n}} d u \int_{u-T / n}^{u} d v \int_{t_{k}^{n}}^{t_{k+1}^{n}} d x \int_{x-T / n}^{x}\left(\frac{\partial^{4} G_{H}}{\partial s^{2} \partial t^{2}}(v, y)+\frac{C_{H}}{(v-y)^{4-2 H}}\right) d y\right| \\
& =\left|C_{H}\right|\left|\int_{t_{j}^{n}}^{t_{j+1}^{n}} d u \int_{u-T / n}^{u} d v \int_{t_{k}^{n}}^{t_{k+1}^{n}} d x \int_{x-T / n}^{x} \frac{d y}{(v+y)^{4-2 H}}\right| \\
& \leqslant\left|C_{H}\right|\left|\int_{t_{j}^{n}}^{t_{j+1}^{n}} d u \int_{u-T / n}^{u} d v \int_{t_{k}^{n}}^{t_{k+1}^{n}} d x \int_{x-T / n}^{x} \frac{d y}{(v-y)^{4-2 H}}\right| \\
& =\left|\rho_{H}(j-k)\right|\left(T n^{-1}\right)^{2 H}
\end{aligned}
$$


for $|j-k| \geqslant 1$. Thus

$$
\left|\widehat{d}_{j, k}^{S^{H}, n}\right| \leqslant 2\left|\rho_{H}(j-k)\right|\left(T n^{-1}\right)^{2 H} \quad \text { for }|j-k| \geqslant 1 .
$$

It still remains to prove the cases when $j=1$ and $k>1$ or $k=1$ and $j>1$. Set $t_{2}^{n, \varepsilon}=t_{2}^{n}-T \varepsilon / n$ and $u^{\varepsilon}=u+T \varepsilon / n$. Since

$$
\begin{aligned}
\left|\widehat{d}_{1 k}^{S^{H}, n}-\widehat{d}_{1 k}^{B^{H}, n}\right|= & \lim _{\varepsilon \rightarrow 0}\left|\int_{t_{1}^{n}}^{t_{2}^{n, \varepsilon}} d u \int_{u^{\varepsilon}-T / n}^{u} d v \int_{t_{k}^{n}}^{t_{k+1}^{n}} d x \int_{x-T / n}^{x}\left(\frac{\partial^{4} G_{H}}{\partial s^{2} \partial t^{2}}(v, y)+\frac{C_{H}}{(v-y)^{4-2 H}}\right) d y\right| \\
\leqslant & \left|C_{H}\right| \lim _{\varepsilon \rightarrow 0}\left|\int_{t_{1}^{n}}^{t_{2}^{n, \varepsilon}} d u \int_{u^{\varepsilon}-T / n}^{u} d v \int_{t_{k}^{n}}^{t_{k+1}^{n}} d x \int_{x-T / n}^{x} \frac{d y}{(v-y)^{4-2 H}}\right| \\
= & \frac{1}{2}\left(T n^{-1}\right)^{2 H} \lim _{\varepsilon \rightarrow 0} \mid[(k+1)-(2-\varepsilon)]^{2 H}-2 \cdot k^{2 H}+(k+1-\varepsilon)^{2 H}-2[k-(2-\varepsilon)]^{2 H} \\
& +4(k-1)^{2 H}-2(k-\varepsilon)^{2 H}+[(k-1)-(2-\varepsilon)]^{2 H}-2(k-2)^{2 H}+(k-1-\varepsilon)^{2 H} \mid \\
= & \left(T n^{-1}\right)^{2 H}\left|\rho_{H}(k-1)\right|
\end{aligned}
$$

then the inequality

$$
\left|\widehat{d}_{1, k}^{S^{H}}\right| \leqslant 2\left|\rho_{H}(k-1)\right|\left(T n^{-1}\right)^{2 H}
$$

holds. A similar argument yields

$$
\left|\widehat{d}_{j, 1}^{S^{H}}\right| \leqslant 2\left|\rho_{H}(j-1)\right|\left(T n^{-1}\right)^{2 H} .
$$

Now we shall prove the statement of the lemma. We will use the estimate (8) and the equalities $\rho_{H}(-r)=\rho_{H}(r), \rho_{H}(0)=4-2^{2 H}$, and $\rho_{H}(1)=-\frac{1}{2}\left(7-4 \cdot 2^{2 H}+3^{2 H}\right)$. Note that for $H \neq 1 / 2$

$$
\begin{aligned}
& \max _{1 \leqslant k \leqslant n-1} \sum_{j=1}^{n-1}\left|d_{j k}^{S^{H}}\right| \leqslant \frac{7}{6}+2 \max _{1 \leqslant k \leqslant n-1} \sum_{\substack{j=1 \\
j \neq k}}^{n-1} \frac{\left|\rho_{H}(j-k)\right|}{\left|\rho_{H}(0)\right|} \\
& \leqslant \frac{7}{6}+4 \sum_{j=1}^{\infty} \frac{\left|\rho_{H}(j)\right|}{\left|\rho_{H}(0)\right|}=\frac{7}{6}+2 \frac{7-4 \cdot 2^{2 H}+3^{2 H}}{4-4^{H}}+4 \sum_{j=2}^{\infty} \frac{\left|\rho_{H}(j)\right|}{\left|\rho_{H}(0)\right|} .
\end{aligned}
$$

In [5] it was proven that

$$
\sum_{j=2}^{\infty} \frac{\left|\rho_{H}(j)\right|}{\left|\rho_{H}(0)\right|}=\frac{1}{2} \cdot \operatorname{sgn}(2 H-1) \frac{3-3 \cdot 2^{2 H}+3^{2 H}}{4-4^{H}}
$$

Thus

$$
\begin{aligned}
\max _{1 \leqslant k \leqslant n-1} \sum_{j=1}^{n-1}\left|d_{j k}^{S^{H}}\right| & \leqslant \frac{7}{6}+2 \frac{7-4 \cdot 2^{2 H}+3^{2 H}}{4-4^{H}}-2 \cdot \operatorname{sgn}(2 H-1) \frac{3-3 \cdot 2^{2 H}+3^{2 H}}{4-4^{H}} \\
& =\left\{\begin{array}{lll}
\frac{7}{6}+2 \frac{10-7 \cdot 4^{H}+2 \cdot 3^{2 H}}{4-4^{H}} & \text { for } H<1 / 2, \\
\frac{7}{6}+2 & \text { for } 1 / 2<H<1
\end{array}\right. \\
& \leqslant\left\{\begin{array}{lll}
\frac{7}{6}+\frac{10}{3} & \text { for } H<1 / 2, \\
\frac{7}{6}+2 & \text { for } 1 / 2<H<1 .
\end{array}\right.
\end{aligned}
$$

\subsection{Bifractional Brownian motion}

Definition 10 (11]) A bifractional Brownian motion (bifBm) $B^{K H}=\left(B_{t}^{K H}, t \geqslant 0\right)$ with parameters $H \in(0,1)$ and $K \in(0,1]$ is a centered Gaussian process with the covariance function

$$
F_{K H}(t, s)=2^{-K}\left(\left(t^{2 H}+s^{2 H}\right)^{K}-|t-s|^{2 H K}\right), \quad s, t \geqslant 0 .
$$

The incremental variance function of bifBm is

$$
\sigma_{B^{H, K}}^{2}(s, t)=\mathbf{E}\left|B_{t}^{H, K}-B_{s}^{H, K}\right|^{2}=2^{1-K}\left[|t-s|^{2 H K}-\left(t^{2 H}+s^{2 H}\right)^{K}\right]+t^{2 H K}+s^{2 H K} .
$$


Let $H \in(0,1)$ and $K \in(0,1]$. Then

$$
2^{-K}|t-s|^{2 H K} \leqslant \sigma_{B, K}^{2}(s, t) \leqslant 2^{1-K}|t-s|^{2 H K}
$$

for all $s, t \in[0, \infty)$ (see [11).

It is known that for bifBm the Orey index is equal to $H K$ (see [13]). If $K=1$ then bifBm becomes $\mathrm{fBm}$, hence we ignore this case.

Lemma 11 Assume that $B^{H, K}=\left\{B^{H, K}(t): t \in[0, T]\right\}$ is a bifBm with $K \in(0,1)$ and $H \in(0,1 / 2)$. Then

$$
\left|\frac{1}{n-1} \sum_{k=1}^{n-1} d_{k k}^{B^{K H}}-1\right| \leqslant\left(\frac{T}{n}\right)^{1 / 2}\left(\frac{1}{6 T} \frac{n}{n-1}+\frac{22}{9 \ln 4}\right),
$$

where

$$
d_{k k}^{B^{K H}}=\frac{n^{2 H}}{2^{1-K} T^{2 H}\left(4-2^{2 H}\right)} \mathbf{E}\left(\Delta_{k, n}^{(2)} B^{H, K}\right)^{2} .
$$

Remark 12 Without this restriction for $H$ the expressions becomes more complicated.

Proof. The proof of the lemma follows the outlines of the proof of Lemma 8 . Observe that the following equality

holds, where

$$
d_{k k}^{B^{K H}}=1-\frac{b(k, H, K)}{4-4^{K H}}
$$

$$
\begin{aligned}
b(k, H, K)= & 2\left[(k+1)^{2 H}+k^{2 H}\right]^{K}+2\left[k^{2 H}+(k-1)^{2 H}\right]^{K} \\
& -2^{K-1}\left[(k+1)^{2 K H}+4 k^{2 K H}+(k-1)^{2 K H}\right]-\left[(k+1)^{2 H}+(k-1)^{2 H}\right]^{K} .
\end{aligned}
$$

By computer modeling we obtain inequalities

$$
\max _{H, K \in(0,1)} d_{k k}^{B^{K H}} \leqslant 1 \quad \text { and } \max _{H, K \in(0,1)} \frac{b(k, H, K)}{4-2^{2 K H}} \leqslant \frac{1}{6}
$$

for all $k \geqslant 1$. Let $\varphi \in \widetilde{\Psi}$ and $\tau_{n}=\left[\varphi\left(T n^{-1}\right) n T^{-1}\right]$. Then

$$
\begin{aligned}
\left|\frac{1}{n-1} \sum_{k=1}^{n-1} d_{k k}^{B K H}-1\right| & \leqslant \frac{1}{n-1} \sum_{k=1}^{\tau_{n}} \frac{b(k, H, K)}{4-2^{2 K H}}+\max _{\tau_{n}+1 \leqslant k \leqslant n-1} \frac{b(k, H, K)}{4-2^{2 K H}} \\
& =I_{1}+I_{2} .
\end{aligned}
$$

and

$$
I_{1} \leqslant \frac{\tau_{n}}{n-1} \max _{1 \leqslant k \leqslant \tau_{n}} \frac{b(k, H, K)}{4-2^{2 K H}} \leqslant \frac{1}{6} \varphi\left(T n^{-1}\right) T^{-1} \frac{n}{n-1} .
$$

Using the inequality $(a+b)^{K} \geqslant 2^{K-1}\left(a^{K}+b^{K}\right), a, b \geqslant 0,0<K<1$, and the formula

$$
(1+x)^{\alpha}=1+\sum_{k=1}^{\infty} \frac{\alpha(\alpha-1) \cdots(\alpha-k+1)}{k !} x^{k}, \quad \text { for }-1<x<1
$$

we obtain

$$
\begin{aligned}
|b(k, H, K)|= & k^{2 H K} \mid 2\left[\left(1+\frac{1}{k}\right)^{2 H}+1\right]^{K}-2^{K-1}\left[\left(1+\frac{1}{k}\right)^{2 K H}+4+\left(1-\frac{1}{k}\right)^{2 K H}\right] \\
& +2\left[1+\left(1-\frac{1}{k}\right)^{2 H}\right]^{K}-\left[\left(1+\frac{1}{k}\right)^{2 H}+\left(1-\frac{1}{k}\right)^{2 H}\right]^{K} \mid \\
\leqslant & 2 k^{2 H K}\left\{2^{1-K}\left[\left(1+\frac{1}{k}\right)^{2 H}+2+\left(1-\frac{1}{k}\right)^{2 H}\right]^{K}\right. \\
& \left.-2^{K-1}\left[\left(1+\frac{1}{k}\right)^{2 K H}+2+\left(1-\frac{1}{k}\right)^{2 K H}\right]\right\} \\
= & 2 k^{2 H K}\left\{2^{1-K}\left[4+\sum_{m=1}^{\infty} \frac{2 H(2 H-1) \cdots(2 H-2 m+1)}{(2 m) !}\left(\frac{1}{k}\right)^{2 m}\right]\right. \\
& \left.-2^{K-1}\left[4+\sum_{m=1}^{\infty} \frac{2 K H(2 K H-1) \cdots(2 K H-2 m+1)}{(2 m) !}\left(\frac{1}{k}\right)^{2 m}\right]\right\}:=\widehat{b}(k, H, K) .
\end{aligned}
$$


Note that the sign of $2 K H(2 K H-1)(2 K H-2) \cdots(2 K H-2 m+1)$ is the same as that of $2 K H-1$. Thus the estimate of $b(k, H, K)$ depends on the signs of $2 H-1$ and $2 K H-1$. Then for $H \in(0,1 / 2)$ and $k \geqslant 2$

$$
\begin{aligned}
\frac{\widehat{b}(k, H, K)}{4-4^{K H}} & \leqslant \frac{2^{K} k^{2 H K}}{4-4^{K H}} \sum_{m=1}^{\infty} \frac{2 K H(1-2 K H) \cdots(2 m-1-2 K H)}{(2 m) !}\left(\frac{1}{k}\right)^{2 m} \\
& \leqslant \frac{2^{K} k^{2 H K}(1-K H)}{4-4^{K H}} \sum_{m=1}^{\infty} \frac{1}{m}\left(\frac{1}{k}\right)^{2 m} \leqslant \frac{2^{K} k^{2 H K}}{\ln 4} \sum_{m=1}^{\infty} \frac{1}{m}\left(\frac{1}{k}\right)^{2 m} \\
& =-\frac{2^{K} k^{2 H K}}{\ln 4} \ln \left(1-\frac{1}{k^{2}}\right) \leqslant \frac{11 \cdot 2^{K}}{9 \ln 4} \frac{1}{k^{2-2 K H}} \leqslant \frac{22}{9 \ln 4} k^{-1}
\end{aligned}
$$

Thus

$$
\left|\frac{1}{n-1} \sum_{k=1}^{n-1} d_{k k}^{B^{K H}}-1\right| \leqslant \frac{1}{6} \varphi\left(T n^{-1}\right) T^{-1} \frac{n}{n-1}+\frac{22}{9 \ln 4} \frac{T}{\varphi\left(T n^{-1}\right) n} .
$$

Let $\varphi\left(T n^{-1}\right)=\left(T n^{-1}\right)^{1 / 2}$. It belongs to the class of functions $\widetilde{\Psi}$. After plugging it into the obtained inequality we get

$$
\begin{aligned}
\left|\frac{1}{n-1} \sum_{k=1}^{n-1} d_{k k}^{B^{K H}}-1\right| & \leqslant \frac{1}{6}\left(\frac{T}{n}\right)^{1 / 2} \frac{n}{T(n-1)}+\frac{22}{9 \ln 4} \frac{T}{\left(T n^{-1}\right)^{1 / 2} n} \\
& \leqslant \frac{1}{6}\left(\frac{1}{n T}\right)^{1 / 2} \frac{n}{n-1}+\frac{22}{9 \ln 4}\left(\frac{T}{n}\right)^{1 / 2}=\left(\frac{T}{n}\right)^{1 / 2}\left(\frac{1}{6 T} \frac{n}{n-1}+\frac{22}{9 \ln 4}\right) .
\end{aligned}
$$

Lemma 13 Assume that $B^{H, K}=\left\{B^{H, K}(t): t \in[0, T]\right\}$ is a bifBm with $K \in(0,1)$ and $H \in(0,1 / 2)$. Then

$$
\max _{1 \leqslant k \leqslant n-1} \sum_{j=1}^{n-1}\left|d_{j k}^{B^{K H}}\right| \leqslant 5.005 .
$$

Proof. The fourth order mixed partial derivative of the covariance function $R^{K H}(s, t)$ has the form

$$
\begin{aligned}
\frac{\partial^{4} F_{K H}}{\partial s^{2} \partial t^{2}}(s, t)= & -\frac{\widehat{C}_{K H}^{(1)}}{|s-t|^{4-2 K H}}+\widehat{C}_{K H}^{(2)}(s t)^{4 H-2}\left(s^{2 H}+t^{2 H}\right)^{K-4} \\
& +\widehat{C}_{K H}^{(3)}(s t)^{2 H-2}\left(s^{2 H}+t^{2 H}\right)^{K-2}
\end{aligned}
$$

for each $s, t>0$ such that $s \neq t$, where

$$
\begin{aligned}
& \widehat{C}_{K H}^{(1)}=2 H K(2 K H-1)(2 H K-2)(2 H K-3) 2^{-K}, \\
& \widehat{C}_{K H}^{(2)}=K(K-1)(K-2)(K-3)(2 H)^{4} 2^{-K}, \\
& \widehat{C}_{K H}^{(3)}=K(K-1)(2 H)^{2}(2 H-1) 2^{-K}(2 K H-2 H-1) .
\end{aligned}
$$

Since $2 s^{H} t^{H} \leqslant s^{2 H}+t^{2 H}$ and $K-2<0, K-4<0$ it follows that

$$
\begin{aligned}
& (s t)^{2 H-2}\left(s^{2 H}+t^{2 H}\right)^{K-2} \leqslant 2^{K-2}(s t)^{K H-2}, \\
& (s t)^{4 H-2}\left(s^{2 H}+t^{2 H}\right)^{K-4} \leqslant 2^{K-4}(s t)^{K H-2} .
\end{aligned}
$$

Let $\widetilde{B}^{K H}$ be a fBm with the Orey index $K H$. Assume that $K H \neq 1 / 2$ and $j \neq 1$ or $k \neq 1$. Using the inequalities 13, 14 and

$$
v y=[y+(v-y)] y \geqslant(v-y) y \geqslant(v-y) \frac{T}{n}, \quad y \geqslant t_{1}^{n},
$$


we obtain

$$
\begin{aligned}
\mid \widehat{d}_{j k}^{B}{ }^{K H} & -2^{1-K} \widehat{d}_{j k}^{\widetilde{B}^{K H}} \mid \\
\leqslant & \left|\widehat{C}_{K H}^{(2)}\right|\left|\int_{t_{j}^{n}}^{t_{j+1}^{n}} d u \int_{u-T / n}^{u} d v \int_{t_{k}^{n}}^{t_{k+1}^{n}} d x \int_{x-T / n}^{x} \frac{d y}{(v y)^{2-4 H}\left(v^{2 H}+y^{2 H}\right)^{4-K}}\right| \\
& +\left|\widehat{C}_{K H}^{(3)}\right|\left|\int_{t_{j}^{n}}^{t_{j+1}^{n}} d u \int_{u-T / n}^{u} d v \int_{t_{k}^{n}}^{t_{k+1}^{n}} d x \int_{x-T / n}^{x} \frac{d y}{(v y)^{2-2 H}\left(v^{2 H}+y^{2 H}\right)^{2-K}}\right| \\
\leqslant & \left|\int_{t_{j}^{n}}^{t_{j+1}^{n}} d u \int_{u-T / n}^{u} d v \int_{t_{k}^{n}}^{t_{k+1}^{n}} d x \int_{x-T / n}^{x} \frac{2^{K-2}\left|\widehat{C}_{K H}^{(2)}\right|+2^{K-4}\left|\widehat{C}_{K H}^{(3)}\right|}{(v y)^{2-K H}} d y\right| \\
\leqslant & \left(\frac{n}{T}\right)^{2-K H}\left|\int_{t_{j}^{n}}^{t_{j+1}^{n}} d u \int_{u-T / n}^{u} d v \int_{t_{k}^{n}}^{t_{k+1}^{n}} d x \int_{x-T / n}^{x} \frac{2^{K-2}\left|\widehat{C}_{K H}^{(2)}\right|+2^{K-4}\left|\widehat{C}_{K H}^{(3)}\right|}{(v-y)^{2-K H}} d y\right| \\
= & \left(\frac{n}{T}\right)^{2-K H} \frac{2^{K-2}\left|\widehat{C}_{K H}^{(2)}\right|+2^{K-4}\left|\widehat{C}_{K H}^{(3)}\right|}{\left|C_{K H}\right|}\left|\widehat{\rho}_{K H}(j-k)\right|\left(T n^{-1}\right)^{K H+2} \\
= & \left(\frac{T}{n}\right)^{2 K H} \frac{2^{K-2}\left|\widehat{C}_{K H}^{(2)}\right|+2^{K-4}\left|\widehat{C}_{K H}^{(3)}\right|}{\left|C_{K H}\right|}\left|\widehat{\rho}_{K H}(j-k)\right|,
\end{aligned}
$$

for $|j-k| \geqslant 1$, where

$$
\begin{aligned}
C_{K H}= & (K H-1) K H(K H+1)(K H+2) \\
\widehat{\rho}_{K H}(j-k)= & 6(j-k)^{K H+2}-4(j-k-1)^{K H+2}-4(j-k+1)^{K H+2} \\
& +(j-k-2)^{K H+2}+(j-k+2)^{K H+2} .
\end{aligned}
$$

So

$$
\left|\widehat{d}_{j k}^{B K H}\right| \leqslant 2^{1-K}\left|\rho_{K H}(j-k)\right|\left(\frac{T}{n}\right)^{2 K H}+\frac{2^{K-2}\left|\widehat{C}_{K H}^{(2)}\right|+2^{K-4}\left|\widehat{C}_{K H}^{(3)}\right|}{\left|C_{K H}\right|}\left|\widehat{\rho}_{K H}(j-k)\right|\left(\frac{T}{n}\right)^{2 K H} .
$$

The cases when $j=1$ and $k>1$ or $k=1$ and $j>1$ can be proven in a way analogous to that of subfBm. Next, we obtain

$$
\max _{1 \leqslant k \leqslant n-1} \sum_{j=1}^{n-1}\left|d_{j k}^{B^{K H}}\right| \leqslant 1+\left(\max _{1 \leqslant k \leqslant n-2}\left|d_{k+1, k}^{B^{K H}}\right|+\max _{2 \leqslant k \leqslant n-1}\left|d_{k-1, k}^{B^{K H}}\right|\right)+\max _{1 \leqslant k \leqslant n-1} \sum_{\substack{j=1 \\|j-k| \geqslant 2}}^{n-1}\left|d_{j k}^{B^{K H}}\right| .
$$

Since

$$
\left|d_{k+1, k}^{B^{K H}}\right| \leqslant \sqrt{d_{k, k}^{B^{K H}} d_{k+1, k+1}^{B^{K H}}}
$$

then by the inequality 11 we get

$$
\max _{1 \leqslant k \leqslant n-2}\left|d_{k+1, k}^{B K H}\right| \leqslant 1 .
$$

Reasoning as in [5], Appendix A we obtain

$$
\sum_{j=2}^{\infty}\left|\widehat{\rho}_{K H}(j)\right|=3-3 \cdot 2^{K H+2}+3^{K H+2} .
$$

Furthermore,

$$
\begin{aligned}
& \max _{1 \leqslant k \leqslant n-1} \sum_{\substack{j=1 \\
|j-k| \geqslant 2}}^{n-1}\left|d_{j k}^{B^{K H}}\right| \\
& \leqslant \max _{1 \leqslant k \leqslant n-1} \sum_{\substack{j=1 \\
|j-k| \geqslant 2}}^{n-1} \frac{\left|\rho_{K H}(j-k)\right|}{4-4^{K H}}+\frac{2^{2 K-3}\left|\widehat{C}_{K H}^{(2)}\right|+2^{2 K-5}\left|\widehat{C}_{K H}^{(3)}\right|}{\left|C_{K H}\right|} \max _{1 \leqslant k \leqslant n-1} \sum_{\substack{j=1 \\
|j-k| \geqslant 2}}^{n-1} \frac{\left|\widehat{\rho}_{K H}(j-k)\right|}{4-4^{K H}} \\
& \leqslant-\frac{1}{2} \operatorname{sgn}(2 K H-1) \frac{3-3 \cdot 2^{2 K H}+3^{2 K H}}{4-4^{K H}}+\frac{2^{2 K-3}\left|\widehat{C}_{K H}^{(2)}\right|+2^{2 K-5}\left|\widehat{C}_{K H}^{(3)}\right|}{\left|C_{K H}\right|} \frac{3-3 \cdot 2^{K H+2}+3^{K H+2}}{4-4^{K H}} .
\end{aligned}
$$


It is clear that

$$
\begin{aligned}
\frac{2^{2 K-3}\left|\widehat{C}_{K H}^{(2)}\right|}{\left|C_{K H}\right|} & =\frac{(1-K)(2-K)(3-K) 2^{4} H^{3} 2^{K-3}}{(1-K H)(K H+1)(K H+2)}=\frac{(H-H K)(2-K)(3-K) 2^{4} H^{2} 2^{K-3}}{(1-K H)(K H+1)(K H+2)} \\
& \leqslant \frac{(2-K)(3-K) H^{2} 2^{K+1}}{(K H+1)(K H+2)} \leqslant \frac{6 \cdot 2^{K-1}}{2} \leqslant 3
\end{aligned}
$$

and

$$
\begin{aligned}
\frac{2^{2 K-5}\left|\widehat{C}_{K H}^{(3)}\right|}{\left|C_{K H}\right|} & =\frac{2^{K-3}(1-K) H|2 H-1|(1+2 H-2 K H)}{(1-K H)(K H+1)(K H+2)} \\
& \leqslant \frac{2^{K-3}|2 H-1|(1+2 H-2 K H)}{(K H+1)(K H+2)} \leqslant \frac{2 \cdot 2^{K-3}}{2} \leqslant \frac{1}{4}
\end{aligned}
$$

Thus

$$
\begin{aligned}
\max _{1 \leqslant k \leqslant n-1} \sum_{j=1}^{n-1}\left|d_{j k}^{B^{K H}}\right| & \leqslant 3+\frac{3-3 \cdot 2^{2 K H}+3^{2 K H}}{2\left(4-4^{K H}\right)}+\frac{13}{4} \cdot \frac{3-3 \cdot 2^{K H+2}+3^{K H+2}}{4-4^{K H}} \\
& \leqslant 3+\frac{1}{4}+\frac{13}{4} \cdot \frac{54}{100}=5,005
\end{aligned}
$$

since the numerator of the second term is a decreasing function, and the numerator of the third term is an increasing function.

\subsection{Ornstein-Uhlenbeck process}

The fractional Ornstein-Uhlenbeck (fO-U) process of the first kind is the unique solution of the stochastic differential equation

$$
X_{t}=x_{0}-\mu \int_{0}^{t} X_{s} d s+\theta B_{t}^{H}, \quad t \leqslant T
$$

with $\mu, \theta>0$, where $B^{H}, 0<H<1$, is a $\mathrm{fBm}$. Its explicit solution is given by

$$
X_{t}=x_{0} e^{-\mu t}+\theta \int_{0}^{t} e^{-\mu(t-u)} d B_{u}^{H}
$$

where the integral exists as a Riemann-Stieltjes integral for all $t>0$ (see, e.g., 6]). First we show the following lemma.

Lemma 14 Let $X$ be the solution of equation $(15)$. Assume that $B^{H}=\left\{B^{H}(t): t \in[0, T]\right\}$ is a $\mathrm{fBm}$ with $H \in\left(0, H^{*}\right]$, where a real number $H^{*}<1$ is known. Then for $H \neq 1 / 2$

$\max _{1 \leqslant k \leqslant n-1} \sum_{j=1}^{n-1}\left|d_{j k}^{X, n}\right| \leqslant \frac{4 \mu T}{4-2^{2 H^{*}}}\left(2 \mu \frac{T}{n}\left[C\left(\frac{T}{n}\right)^{2-2 H^{*}}+1\right]+\sqrt{2\left(4-2^{2 H^{*}}\right)}\left[C\left(\frac{T}{n}\right)^{2-2 H^{*}}+1\right]^{1 / 2}\right)+\frac{8}{3}$,

where $C=\mu^{2}\left[3 x_{0}^{2} \theta^{-2}+6 T^{2}\right]$ and

$$
d_{j k}^{X, n}=\frac{n^{2 H}}{\theta^{2} T^{2 H}\left(4-2^{2 H}\right)} \widehat{d}_{j k}^{X, n}, \quad \widehat{d}_{j k}^{X, n}=\mathbf{E}\left(\Delta_{n, j}^{(2)} X \Delta_{n, k}^{(2)} X\right) .
$$

Proof. Denote

$$
\Delta_{n, k} Z=-\mu \int_{t_{k-1}^{n}}^{t_{k}^{n}} X_{s} d s
$$

It is clear that

$$
\begin{aligned}
& \left|\widehat{d}_{j k}^{X, n}-\theta^{2} \widehat{d}_{j k}^{B^{H}, n}\right|=\mid \mathbf{E}\left[\Delta_{n, j+1} Z-\Delta_{n, j} Z\right]\left[\Delta_{n, k+1} Z-\Delta_{n, k} Z\right]+\theta \mathbf{E}\left[\Delta_{n, j+1} Z-\Delta_{n, j} Z\right] \Delta_{n, k}^{(2)} B^{H} \\
& +\theta \mathbf{E}\left[\Delta_{n, k+1} Z-\Delta_{n, k} Z\right] \Delta_{n, j}^{(2)} B^{H}
\end{aligned}
$$

and

$$
\left[\Delta_{n, j+1} Z-\Delta_{n, j} Z\right]=-\mu\left[\int_{t_{j}^{n}}^{t_{j+1}^{n}}\left[X_{s}-X_{j}\right] d s-\int_{t_{j-1}^{n}}^{t_{j}^{n}}\left[X_{s}-X_{j}\right] d s\right]
$$


Reasoning as in 13 , we obtain

$$
\sup _{t \leqslant T} \mathbf{E} X_{t}^{2} \leqslant 3 x_{0}^{2}+6 \theta^{2} T^{2}
$$

and

$$
\begin{aligned}
\mathbf{E}\left[X_{t}-X_{j}\right]^{2} & \leqslant 2 \mu^{2} \frac{T}{n} \int_{t_{j}^{n}}^{t} \mathbf{E} X_{s}^{2} d s+2 \theta^{2}\left(\frac{T}{n}\right)^{2 H} \leqslant 2 \mu^{2}\left[3 x_{0}^{2}+6 \theta^{2} T^{2}\right]\left(\frac{T}{n}\right)^{2}+2 \theta^{2}\left(\frac{T}{n}\right)^{2 H} \\
& =2 \theta^{2}\left(\frac{T}{n}\right)^{2 H}\left[C\left(\frac{T}{n}\right)^{2-2 H}+1\right]
\end{aligned}
$$

where $C=\mu^{2}\left[3 x_{0}^{2} \theta^{-2}+6 T^{2}\right]$. Thus

$$
\begin{aligned}
\left|\widehat{d}_{j k}^{X, n}-\theta^{2} \widehat{d}_{j k}^{B H}, n\right| \leqslant & 8 \mu^{2} \theta^{2}\left(\frac{T}{n}\right)^{2+2 H}\left[C\left(\frac{T}{n}\right)^{2-2 H}+1\right] \\
& +4 \sqrt{2\left(4-2^{2 H}\right)} \mu \theta^{2}\left(\frac{T}{n}\right)^{1+2 H}\left[C\left(\frac{T}{n}\right)^{2-2 H}+1\right]^{1 / 2} .
\end{aligned}
$$

Consequently,

$$
\begin{aligned}
& \max _{1 \leqslant k \leqslant n-1} \sum_{j=1}^{n-1}\left|d_{j k}^{X, n}\right| \leqslant \max _{1 \leqslant k \leqslant n-1} \sum_{j=1}^{n-1}\left|d_{j k}^{X, n}-\theta^{2} d_{j k}^{B^{H}, n}\right|+\max _{1 \leqslant k \leqslant n-1} \sum_{j=1}^{n-1}\left|d_{j k}^{B^{H}, n}\right| \\
\leqslant & \frac{8 \mu^{2}}{4-2^{2 H^{*}}} \frac{T^{2}}{n}\left[C\left(\frac{T}{n}\right)^{2-2 H^{*}}+1\right]+\frac{4 \sqrt{2} \mu T}{\sqrt{4-2^{2 H^{*}}}}\left[C\left(\frac{T}{n}\right)^{2-2 H^{*}}+1\right]^{1 / 2}+\frac{8}{3} \\
= & \frac{4 \mu T}{4-2^{2 H^{*}}}\left(2 \mu \frac{T}{n}\left[C\left(\frac{T}{n}\right)^{2-2 H^{*}}+1\right]+\sqrt{2\left(4-2^{2 H^{*}}\right)}\left[C\left(\frac{T}{n}\right)^{2-2 H^{*}}+1\right]^{1 / 2}\right)+\frac{8}{3} .
\end{aligned}
$$

Lemma 15 Let $X$ be the solution of the equation (15). Then

$$
\begin{aligned}
\left|\frac{1}{n-1} \sum_{k=1}^{n-1} d_{k k}^{X, n}-1\right| \leqslant & \frac{4 \mu}{4-2^{2 H^{*}}} \frac{T}{n}\left(2 \mu \frac{T}{n}\left[C\left(\frac{T}{n}\right)^{2-2 H^{*}}+1\right]\right. \\
& \left.+\sqrt{2\left(4-2^{2 H^{*}}\right)}\left[C\left(\frac{T}{n}\right)^{2-2 H^{*}}+1\right]^{1 / 2}\right) .
\end{aligned}
$$

Proof. To prove this lemma, observe that

$$
\begin{aligned}
\left|\frac{1}{n-1} \sum_{k=1}^{n-1} d_{k k}^{X, n}-1\right| & \leqslant \max _{1 \leqslant k \leqslant n-1}\left|d_{k k}^{X, n}-d_{k k}^{B^{H, n}}\right|+\left|\frac{1}{n-1} \sum_{k=1}^{n-1} d_{k k}^{B^{H, n}}-1\right| \\
& =\max _{1 \leqslant k \leqslant n-1}\left|d_{k k}^{X, n}-1\right| .
\end{aligned}
$$

From the inequality

$$
\begin{aligned}
\left|\widehat{d}_{k k}^{X, n}-\theta^{2} \widehat{d}^{B^{H}, n}\right| \leqslant & 8 \mu^{2} \theta^{2}\left(\frac{T}{n}\right)^{2+2 H}\left[C\left(\frac{T}{n}\right)^{2-2 H}+1\right] \\
& +4 \sqrt{2\left(4-2^{2 H}\right)} \mu \theta^{2}\left(\frac{T}{n}\right)^{1+2 H}\left[C\left(\frac{T}{n}\right)^{2-2 H}+1\right]^{1 / 2}
\end{aligned}
$$

it follows that the statement of the lemma holds.

\section{Simulations}

The simulations of the obtained confidence intervals presented below were performed using the $\mathrm{R}$ software environment [16. Sample paths of $\mathrm{fBm}$ were generated using the circulant matrix embedding method and were further used to simulate the sample paths of the fractional Ornstein-Uhlenbeck process (15). The constants for the latter were (arbitrarily) chosen as $x_{0}=0$ and $\mu=0.5$. Sample paths of the sub-fractional and bifractional Brownian motion 
were simulated using the Cholesky method. Due to the notable computational requirements of this method the maximum sample path length considered was $n=1600$. Figures presented below correspond to the case of the confidence level $1-\alpha, \alpha=0.1$. The observed coverage percentages in all cases were at least as good as claimed in Theorem 6 .

Figures 1-5 present the confidence interval (CI) lengths for all the process types considered in this paper. Figure 6 shows the median ratios of the confidence intervals lengths, where the $\mathrm{CI}$ lengths of the subfBm, bifBm and fO-U processes were divided by the corresponding CI lengths of fBm. It can be seen that in almost all cases the confidence intervals behave in a similar way, one notable exception being the case of fO- $U$ as the value of $H$ approaches 1 . This is hardly unexpected given the normalization used in Lemma 14 and in this scenario the CI covers the whole interval of possible parameter values $0<H<1$.

fBm Cl length (alpha=0.1)

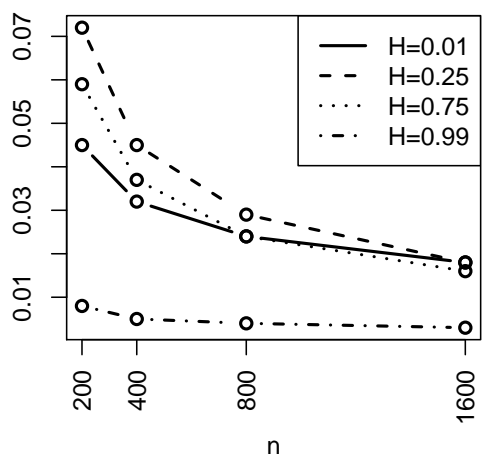

Figure 1: $\left|H_{n}^{\sup }(\alpha)-H_{n}^{i n f}(\alpha)\right|$

\section{subfBm Cl length (alpha=0.1)}

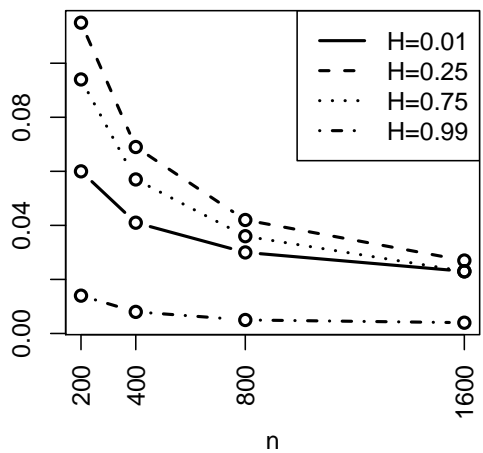

Figure 2: $\left|H_{n}^{\text {sup }}(\alpha)-H_{n}^{\text {inf }}(\alpha)\right|$

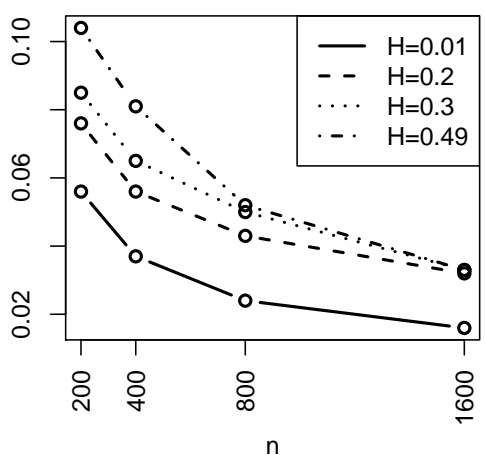

Figure 3: $\left|(H K)_{n}^{\text {sup }}(\alpha)-(H K)_{n}^{\text {inf }}(\alpha)\right|$
bifBm Cl length (alpha=0.1 ,K=0.9)

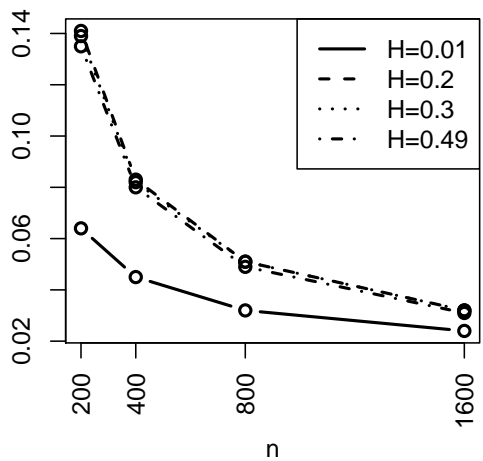

Figure 4: $\left|(H K)_{n}^{\text {sup }}(\alpha)-(H K)_{n}^{\text {inf }}(\alpha)\right|$ 


\section{O-U Cl length (alpha $=0.1)$}

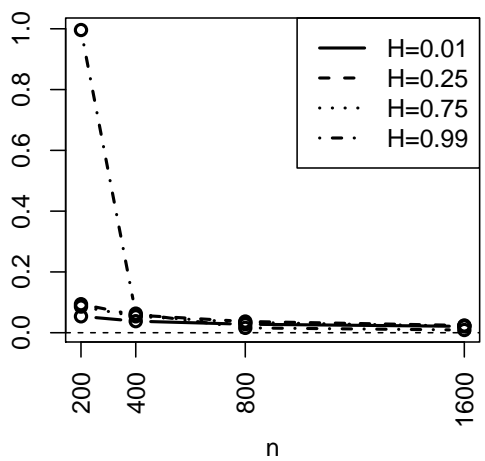

Figure 5: $\left|H_{n}^{\text {sup }}(\alpha)-H_{n}^{\text {inf }}(\alpha)\right|$
Ratios of $\mathrm{Cl}$ lengths

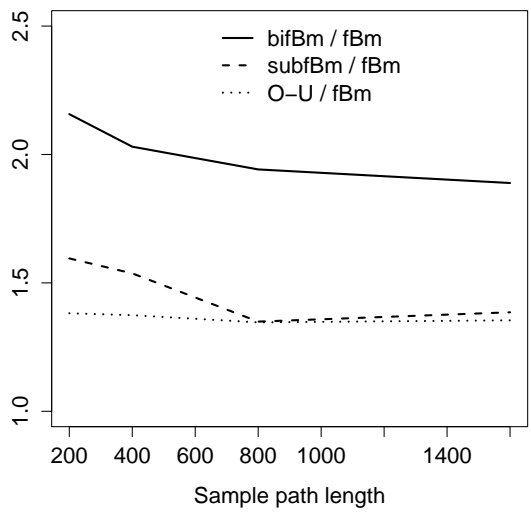

Figure 6: Comparison of CI lengths

\section{References}

[1] A. Bégyn, Quadratic variations along irregular partitions for Gaussian processes, Electronic Journal of Probability, 10 (2005), 691-717.

[2] A. Bégyn, Asymptotic development and central limit theorem for quadratic variations of Gaussian processes, Bernoulli, 13(3) (2007), 712-753.

[3] T. Bojdecki, L. G. Gorostiza, A. Talarczyk, Sub-fractional Brownian motion and its relation to occupation time, Stat. \& Probab. Lett. 69 (2004), 405-419.

[4] Breton J-C, Nourdin I, Peccati G., Exact confidence intervals for the Hurst parameter of a fractional Brownian motion. Electron J. Stat. 3, 416425 (2009).

[5] Breton J-C, J.-F. Coeurjolly, Confidence intervals for the Hurst parameter of a fractional Brownian motion based on finite sample size, Stat Inference Stoch Process (2012) 15, 126.

[6] P. Cheridito, H. Kawaguchi, and M. Maejima, Fractional Ornstein-Uhlenbeck processes. Electronic Journal of Probability, 8, p. 1-14 (2003).

[7] J.-F. Coeurjolly, Estimating the parameters of a fractional Brownian motion by discrete variations of its sample paths, Statistical Inference for Stochastic Processes, 4 (2001), 199-227.

[8] A. Benassi, S. Cohen, J. Istas, and S. Jaffard, Identification of filtered white noises, Stochastic Processes and their Applications, 75 (1998), 31-49.

[9] Gladyshev, E. G., A new Limit theorem for stochastic processes with Gaussian increments, Theory Probab. Appl., 6(1), p.52-61, (1961).

[10] X. Guyon and J. Léon. Convergence en loi des H-variations d'un processus gaussien stationnaire sur R. Ann. Inst. Poincaré, 25, p. 265-282 (1989).

[11] C. Houdré and J. Villa, An example of infinite dimensional quasi-helix, Contemporary Mathematics 366 (2003), 195-201.

[12] J. Istas, G. Lang. Quadratic variations and estimation of the local H older index of a Gaussian process. Ann. Inst. Henri Poincaré, Probab. Stat., 33, p. 407-436 (1997).

[13] K. Kubilius, On estimation of the extended Orey index for Gaussian processes. To appear in Stochastics An International Journal of Probability and Stochastic Processes.

[14] J. Liu, L. Yan, Z. Peng, and D. Wang, Remarks on confidence intervals for self-similarity parameter of a subfractional Brownian motion, Abstract and Applied Analysis, 2012, Article ID 804942, 14 pages (2012).

[15] R. Malukas, Limit theorems for a quadratic variation of Gaussian processes, submited to Nonlinear Analysis: Modelling and Control. 
[16] R Core Team (2014), R: A language and environment for statistical computing. R Foundation for Statistical Computing, Vienna, Austria. URL http://www.R-project.org/.

[17] R. Norvaiša, A coplement to Gladyshev's theorem, Lith. Math. J., 51(1), 26-35 (2011).

[18] R. Norvaiša, Gladyshev's theorem for integrals with respect to a Gaussian process, Preprint, 2011.

\section{Appendix. Code listings}

\section{genFBM.r}

$\#$ genFBM returns a single sample path of the fBm \#
$\#-$ the Hurst index
$\#-$ length of the sample path
\#

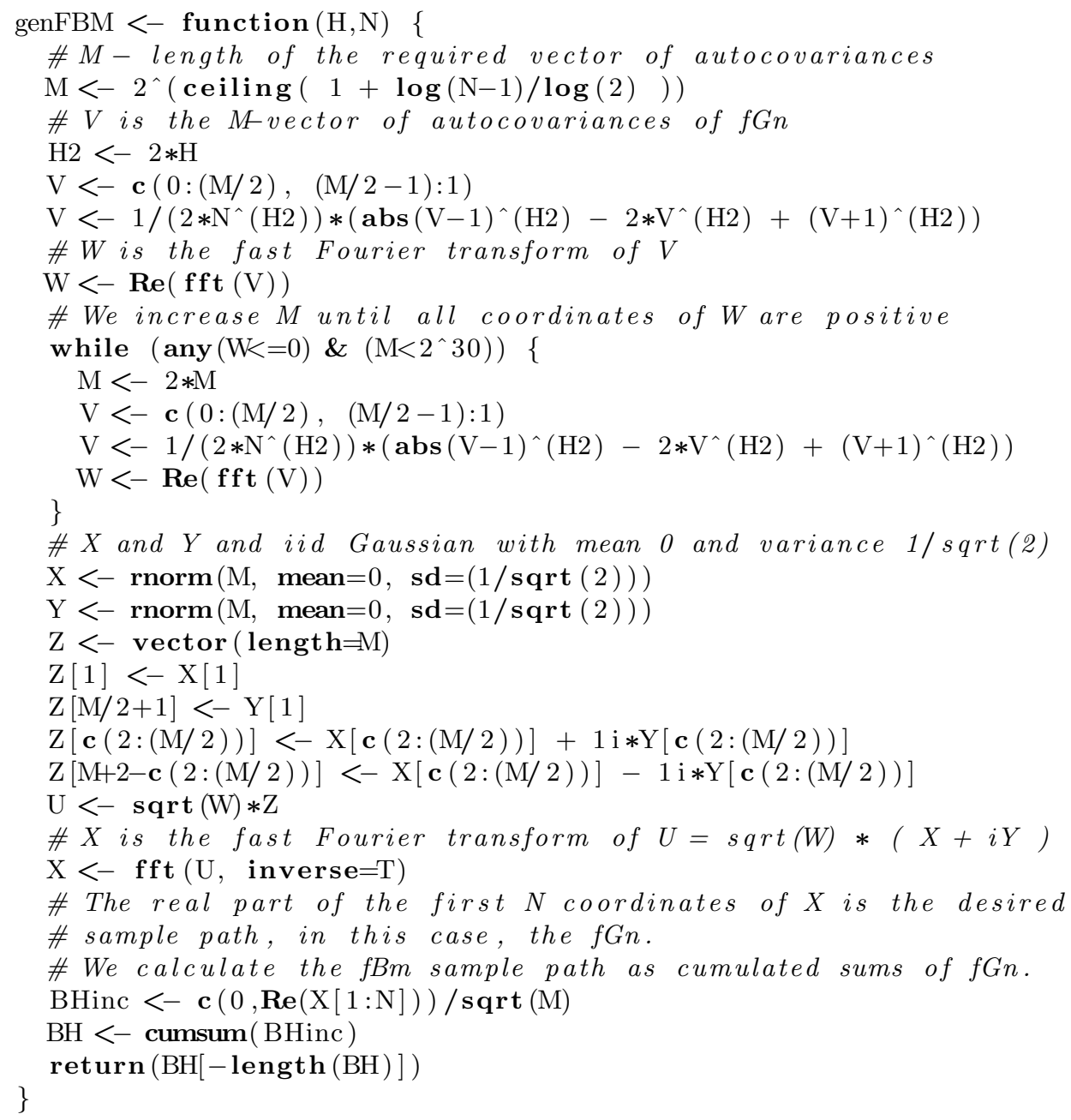

\section{Cholesky.r}

$\#$ genSFBMch generates a batch of sample paths \#
$\#$ of sub-fractional Brownian motion
$\# H-$ the Hurst index
$\# N-$ length of sample paths




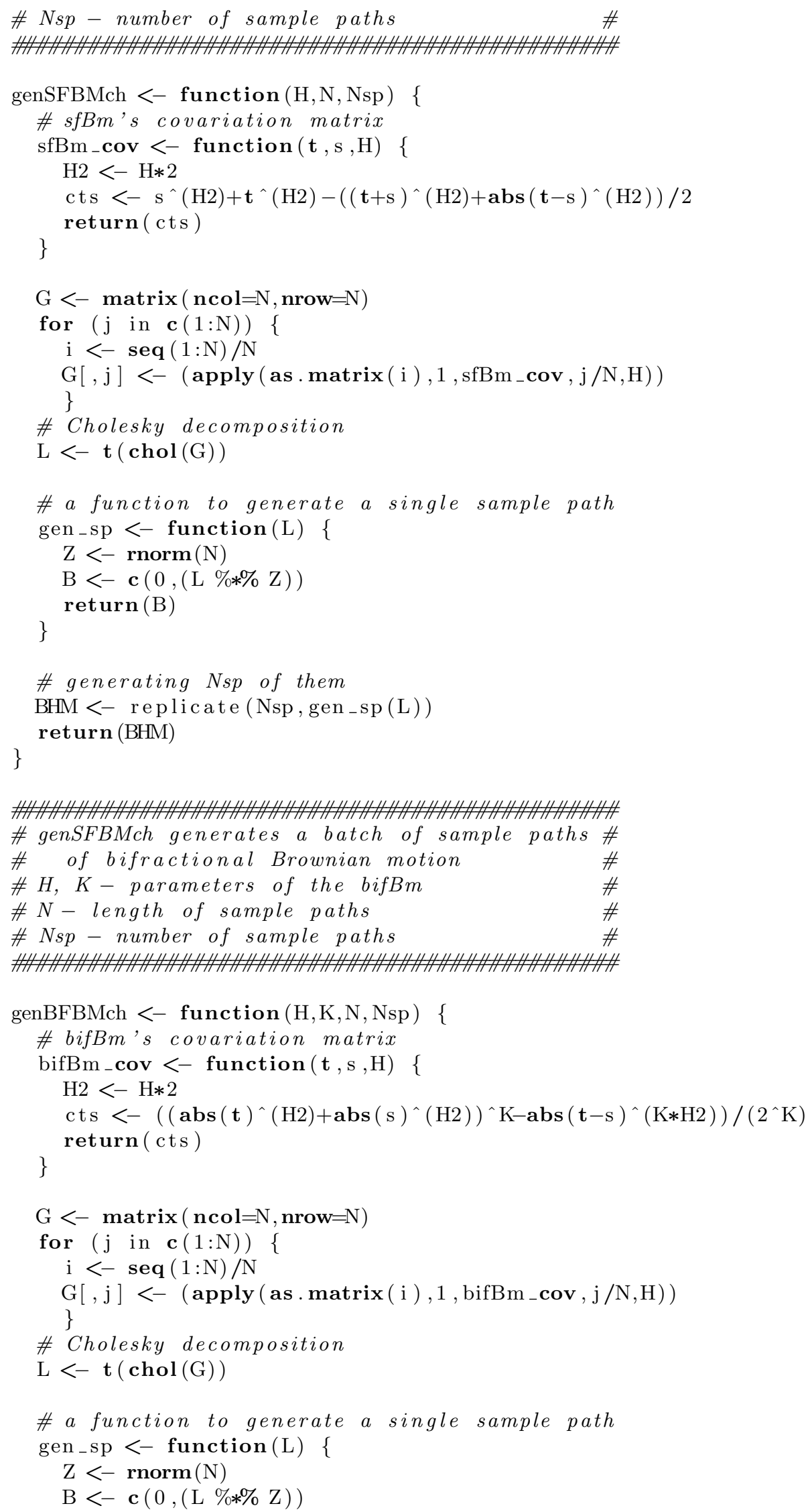




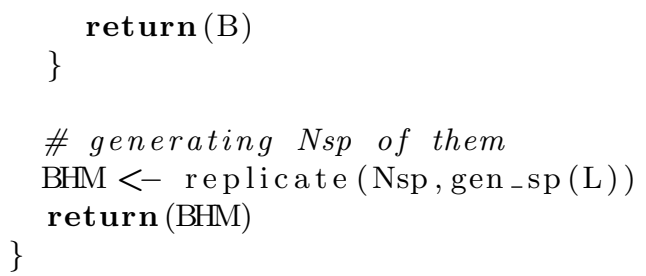

\section{fbmCI.r}

The following code evaluates the confidence intervals for the fractional Brownian motion. Simulations for other processes considered in this paper were performed in a similar way.

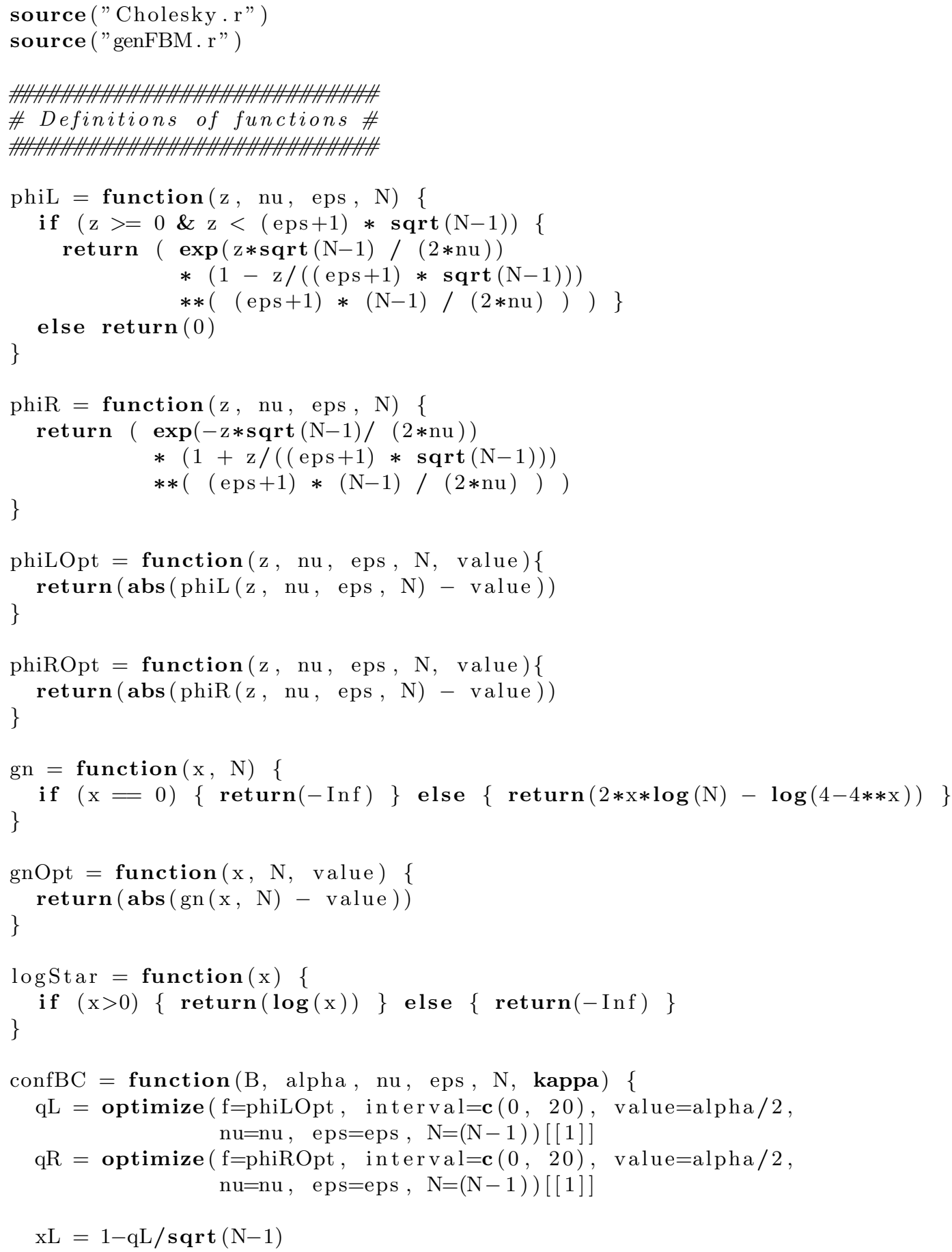




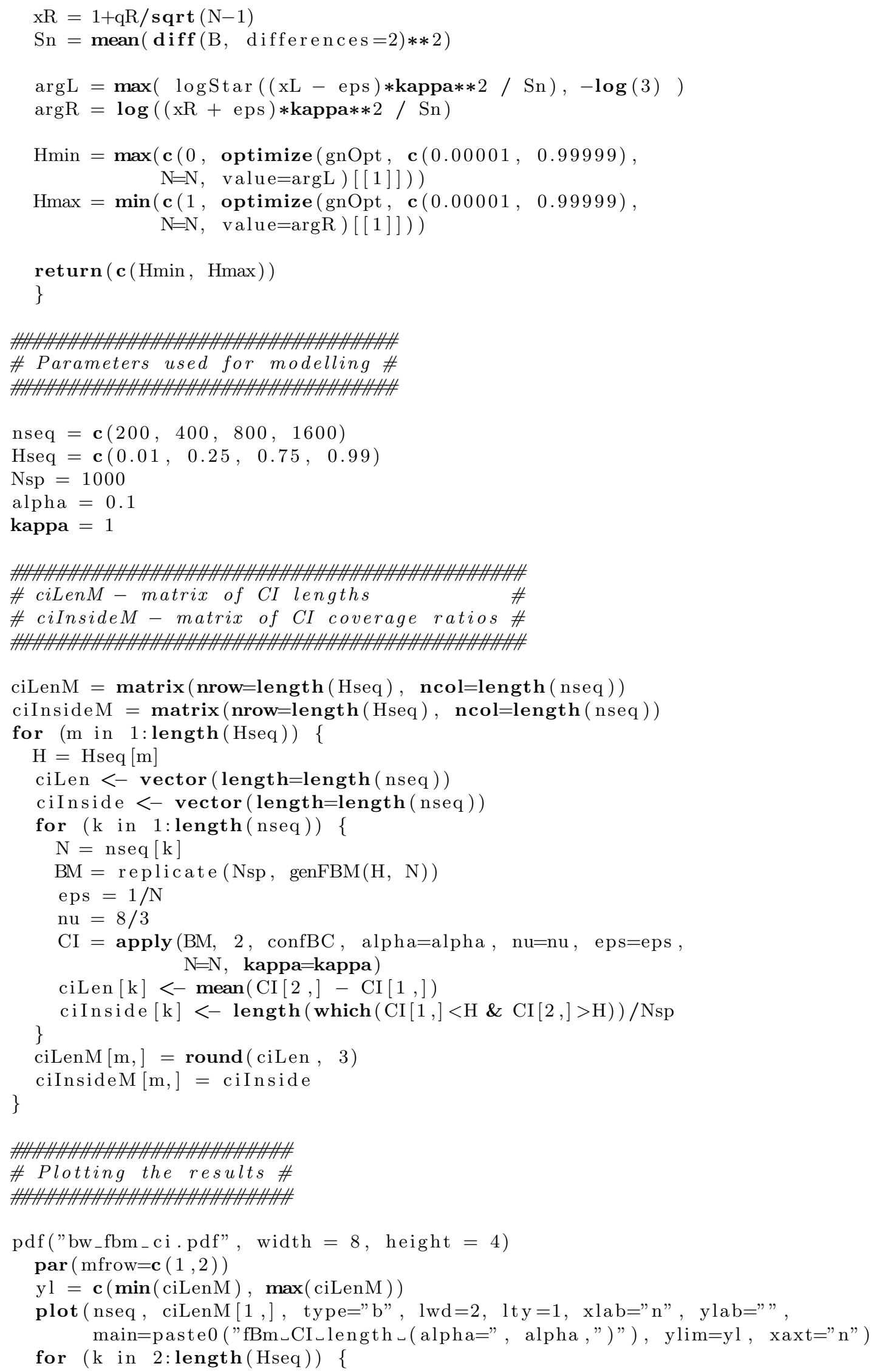




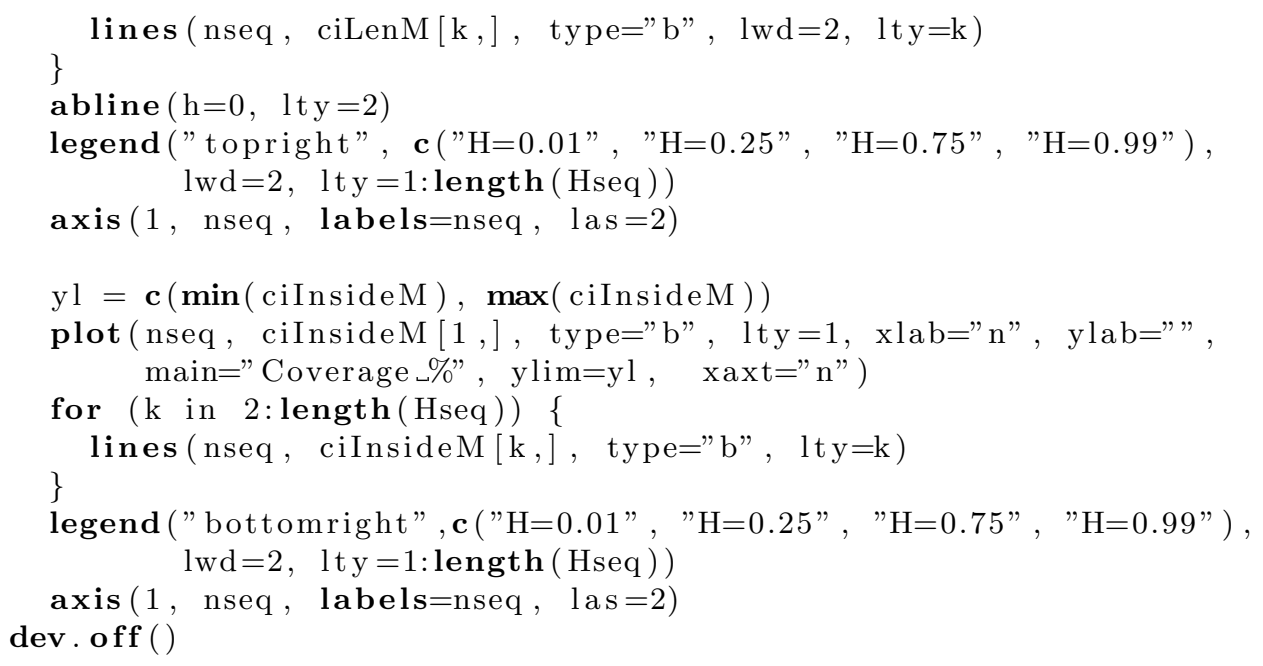

